\title{
Simulation of a suite of generic long-pulse neutron instruments to optimize the time structure of the ESS
}

Kim Lefmann, ${ }^{1,2}$ Kaspar H. Klen $\varnothing,{ }^{1,2}$ Jonas Okkels Birk, ${ }^{1,2,3}$ Britt R. Hansen, ${ }^{4,5}$ Sonja L. Holm, ${ }^{1,2}$ Erik Knudsen, ${ }^{4,5}$ Klaus Lieutenant, ${ }^{6,7,8}$ Lars von Moos, ${ }^{9,5,10}$ Morten Sales, ${ }^{1,2}$ Peter K. Willendrup, ${ }^{4,5}$ and Ken H. Andersen ${ }^{11}$

1) Nanoscience and eScience Centers, Niels Bohr Institute, University of Copenhagen, Universitetsparken 5, 2100 Copenhagen Ø, Denmark

2) Danish Workpackage for the ESS Design Update Phase, Universitetsparken 5, 2100 Copenhagen $\varnothing$, Denmark

3) Laboratory for Quantum Magnetism, École Polytecnique Fédérale de Lausanne (EPFL), 1015 Lausanne, Switzerland

4) Institute of Physics, Technical University of Denmark, 2800 Lyngby, Denmark

5) Danish Workpackage for the ESS Design Update Phase, 2800 Lyngby, Denmark

6) Institute for Energy Technology, Instituttveien 18, 2007 Kjeller, Norway

7) Helmholtz Center for Energy and Materials, Hahn-Meitner Platz, 14109 Berlin, Germany

8) German Work Package for the ESS Design Update, Hahn-Meitner Platz, 14109 Berlin, Germany

9) Department of Energy Conversion and Storage, Technical University of Denmark, 4000 Roskilde, Denmark

10) Institute for Energy Conversion, Technical University of Denmark, 4000 Roskilde, Denmark

${ }^{11)}$ European Spallation Source ESS AB, 22100 Lund, Sweden

We here describe the result of simulations of 15 generic neutron instruments for the long-pulsed European Spallation Source (ESS). All instruments have been simulated for 20 different settings of the source time structure, corresponding to pulse lengths between $1 \mathrm{~ms}$ and $2 \mathrm{~ms}$; and repetition frequencies between $10 \mathrm{~Hz}$ and $25 \mathrm{~Hz}$. The relative change in performance with time structure is given for each instrument, and an unweighted average is calculated. The performance of the instrument suite is proportional to a) the peak flux and b) the duty cycle to a power of approx. 0.3. This information is an important input to determining the best accelerator parameters. In addition, we find that in our simple guide systems, most neutrons reaching the sample originate from the central $3-5 \mathrm{~cm}$ of the moderator. This result can be used as an input in later optimization of the moderator design. We discuss the relevance and validity of defining a single Figure-ofMerit for a full facility and compare with evaluations of the individual instrument classes.

\section{INTRODUCTION}

The European Spallation Source (ESS) is designed to be a long-pulsed spallation neutron source - the first of its kind $^{1,2}$. This opens new territory, including the challenges to design instruments that perform well for a longpulsed source, to design the optimal moderator for these instruments, and to choose the pulsing time structure that matches these choices. Obviously, these optimizations are coupled, since e.g. the instrument design depends upon the pulse length and the optimal moderator design depends on both desired pulse length and on the instrument geometries.

In this article we are concerned with only one part of this optimization problem: the selection of the source time structure, i.e. its pulse length $(\tau)$ and repetition time $(T)$. The original 2002 design was fixed at $\tau=2 \mathrm{~ms}$, and $T=60 \mathrm{~ms}\left(f=16 \frac{2}{3} \mathrm{~Hz}\right)^{3}$, and we have therefore investigated time structures in the neighbourhood of these initial parameters.

In order to perform the time-structure optimization, we have selected a suite of generic instruments, covering a broad range of scientific utilizations. These instruments have then undergone a rough design and optimization for each setting of $(T, \tau)$, and the relative merits of the instruments at the different time structures have been compiled and compared.

The simulated instrument suite should not be seen as a draft day-one suite, neither should the individual instruments be seen as being close to their final design. Much design work and careful selection of an initial instrument suite is presently in progress. The present work is merely the first step in a long process.

Below, we present our generic neutron long-pulse instrument suite, the optimization procedure, and the obtained overall results. The simulation results of the 15 individual instruments are available on-line ${ }^{4}$ and are or will be published individually in more detailed articles ${ }^{5-12}$.

As a result of this and other studies of the ESS time structure, covering its impact on the performance, reliability, construction cost and operation of the facility, the time structure has now been fixed at $\tau=2.86 \mathrm{~ms}$ and $T=71 \mathrm{~ms}(f=14 \mathrm{~Hz})$. The results presented in this paper were an important input to this decision. 


\begin{tabular}{|l|lll|}
\hline Instrument & $L_{1}[\mathrm{~m}]$ & $L_{2}[\mathrm{~m}]$ & $\beta$ \\
\hline Cold Chopper Spect. & 60 & 100 & 0 \\
Therm. Chopper Spect. & 100 & 100 & 0 \\
Cold Triple Axis & 40 & 40 & 0 \\
Thermal Triple Axis & 40 & 40 & 0 \\
TOF Triple Axis & 60 & 100 & 0 \\
Backscatter Spectrometer & 151 & 302 & 0 \\
\hline Spin Echo Spectrometer & 30 & 30 & 2.5 \\
\hline Short SANS (bio-) & \multicolumn{1}{|c|}{$12+1-4$} & 2.5 \\
Medium SANS & $18+1-10$ & 2.5 \\
Long SANS (materials-) & $28+2-20$ & 2.5 \\
\hline Horizontal Reflectometer & 52 & 52 & 4 \\
Vertical Reflectometer & 52 & 52 & 4 \\
\hline Cold Powder Diffract. & 88 & 176 & 0 \\
Thermal Powder Diffract. & 102 & 102 & 0 \\
Single Crystal Diffract. & 31 & 42 & 0 \\
\hline
\end{tabular}

TABLE I. Properties of 15 generic ESS instruments, suggested by the ESS-S SAG and the ESS SAC. $L_{1}$ denotes the length of the instrument for a pulse length of $\tau=1 \mathrm{~ms}$, while $L_{2}$ is the instrument length for $\tau=2 \mathrm{~ms}$, and $\beta$ is the "Frascati exponent", defined by (1).

\section{THE GENERIC INSTRUMENT SUITE}

The instrument suite we discuss here was initiated by the Scientific Advisory Group (SAG) for ESSScandinavia, in September 2009. This list was expanded by the slightly different "straw-man-list" of instruments, decided upon by the Scientific Advisory Council for the ESS (SAC) in June 2010. Our starting list was found as a join of these two instrument suites, and is shown in Table I. It should be noted that due to time constraints, neither a tomography instrument, a protein diffractometer, nor a wide-angle spin-echo instrument have been included in these simulations, even though these classes of instruments were present in the straw-man suite.

In the optimizations, we have taken into account that neutrons of different wavelengths may not be equally useful for the individual instruments. In particular, spin-echo spectrometers, reflectometers, and small-angle diffractometers strongly prefer long wavelength neutrons. To account for this fact in a simplified way, we parametrize the relative "value", $V$, of each neutron by a simple expression:

$$
V(\lambda)=\lambda^{\beta}
$$

The values of $\beta$ for different instrument types were selected by an expert meeting in Frascati, August $2009^{13}$, and Table I contains the chosen values of $\beta$. Here, a value of zero indicates that all neutrons are considered equally valuable, while a positive value of $\beta$ gives preference to long-wavelength neutrons.

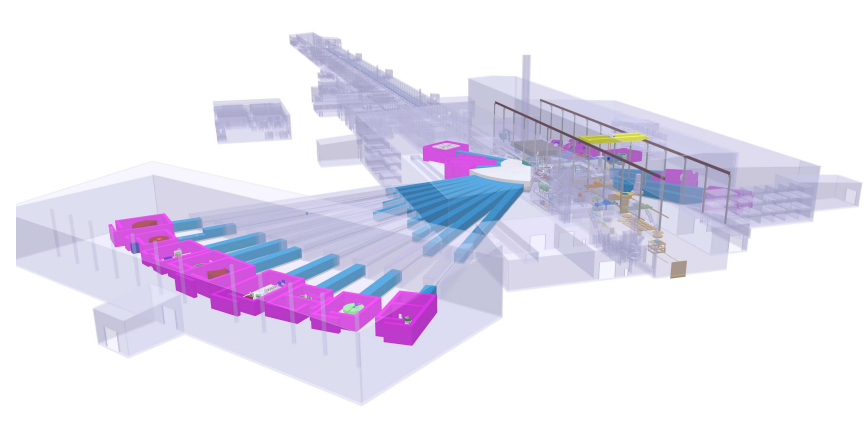

FIG. 1. (Color online) Artists view of the ESS target/instrument buildings seen obliquely from above. Note that the long instruments are placed in a hall (foreground left) which is separated from the main target building (right). The accelerator is seen stretching into the background.

\section{DESIGN AND OPTIMIZATION OF INSTRUMENTS}

Over the last decade or more, a number of authors have addressed the issue of long-pulse instrumentation ${ }^{14-17}$. The instrument concepts and designs simulated here are in general adapted from the earlier work, except that we have adjusted the instrument lengths as described below and listed in Table I. Most instruments on this list are typical time-of-flight instruments, except the reactor-type triple-axis instruments. One untraditional instrument type, labeled "TOF Triple Axis" has been included in the list. This is a hybrid (or invertedgeometry) spectrometer ${ }^{11}$ with a time-of-flight front-end and a triple-axis-like crystal analyzer back-end.

\section{A. Instrument length and resolution}

To qualify the discussion, let us first recall the equation for the neutron time-of-flight, $t$ :

$$
t=\alpha \lambda L,
$$

where $L$ is the flight length and $\alpha=m_{\mathrm{n}} / h \approx 252.7 \mu \mathrm{s} /(\mathrm{m}$ $\AA)$. The relative uncertainty of the neutron wavelength can then be expressed by the uncertainty in flight time by

$$
\frac{\delta \lambda}{\lambda}=\frac{\delta t}{t}=\frac{\delta t}{\alpha \lambda L}
$$

For long-pulse instruments, $\delta t$ is either given approximately by the pulse length, $\tau$, (at a long pulsed source, the exponentially decaying tail of the pulse can to first order be neglected compared to $\tau$ ), or by the opening time of a pulse-defining chopper, as described below. In the latter case, $L$ will be the flight length from the pulsedefining chopper to the detector, in the former it will denote the full instrument length to the detector (for chopper spectrometers, see later). 
In analogy, the useful wavelength band, $\Delta \lambda$, of neutrons which can reach the detector without creating frame overlap is given by

$$
\Delta \lambda=\frac{\Delta t}{\alpha L},
$$

where for instruments using the full pulse, $\Delta t=T-\delta t \approx$ $T$.

A number of the simulated instruments cannot directly utilize the full pulse length, $\tau$, since this would result in a too bad resolution, (too large $\delta \lambda / \lambda$ ). Therefore, pulse shaping must be performed at a fast pulse-defining chopper, close to the source. In this work, the distance between source and chopper is set to the smallest realistic value given by the biological shielding of the moderator: $L_{\mathrm{pc}}=6 \mathrm{~m}$.) A pulse-defining chopper at the distance $L_{\mathrm{pc}}$ effectively selects a wavelength band, given by $\Delta \lambda=$ $\tau /\left(\alpha L_{\mathrm{pc}}\right)$. To let this wavelength band fill the whole time frame, $T$, at the detector, the instrument must be very long: $L=L_{\mathrm{pc}}(1+T / \tau)$, which for the parameters investigated in this work lies between $126 \mathrm{~m}$ and $606 \mathrm{~m}$, since the inverse duty cycle, $T / \tau$, lies in the range 20-100.

\section{B. Wavelength Frame Multiplication and Repetition Rate Multiplication}

At some instruments with pulse-defining choppers, we have used an alternative scheme to having very long instruments: A number of closely-spaced shorter pulses is produced at the pulse-defining chopper, which are then kept separated by a number of sub-frame-overlap choppers. This has been denoted "Wavelength Frame Multiplication" (WFM), as first presented by the group of F. Mezei ${ }^{18,19}$. In the present simulations, the WFM method is used at the thermal powder diffractometer and the thermal chopper spectrometer.

The cold chopper spectrometer uses a similar technique, which bears the name "Repetition Rate Multiplication" (RRM). Here, the full pulse length is used, but a monochromating chopper close to the sample produces up to 15 different monochromatic pulses for each moderator pulse ${ }^{14,20}$, as simulated in Ref. ${ }^{5}$. Recently, this technique has been experimentally proven feasible at NEAT, $\mathrm{HZB}^{21}$ and 4 SEASONS, J-PARC ${ }^{22}$. In the present simulations, also the thermal chopper spectrometer employs RRM (in addition to using WFM).

\section{The source}

Lacking precise information about the source power and moderator performance for the different time structures, we have initially considered the two following scenarios.

1. The source has a constant time-average neutron flux.
2. The accelerator is limited by a maximum beam current; i.e. the source peak flux is constant.

These two scenarios differ only by a $\tau / T$ scaling of the source flux, whence we were able to use the same set of simulations/optimizations. As a reference point at the baseline settings, we use the characteristics of a $12 \times 12 \mathrm{~cm}^{2}$ moderator with uniform flux distribution, as given in Ref. 23 .

\section{The guide systems}

For the short guide systems (below $60 \mathrm{~m}$ ), we have everywhere used guides with constant cross section, where fast-neutron background from direct line-of-sight to the moderators is avoided by inserting a kink or curved section. At the reflectometers, we have used elliptical focusing in the direction perpendicular to the sample surface, combined with a kink in the other direction.

For instruments of $60 \mathrm{~m}$ and longer, and for the $40 \mathrm{~m}$ triple-axis instruments, we have employed elliptical guides for beam transport, since recent experiments and simulations has shown this design to be strongly superior over traditional curved guides ${ }^{6,24}$.

For the values of guide reflectivities, we have everywhere used recent information from one supplier ${ }^{25}$. In general, we use $m=3$ along the main length of all guides, and $m=6$ in the beginning and end of elliptical guides.

Guides have everywhere been assumed to consist of straight sections, with perfect alignment and zero waviness. The effect of waviness and misalignment of (in particular) long elliptical guides is a topic of future simulations ${ }^{26}$. A similar work was carried out earlier for straight guide geometries ${ }^{27}$.

In the optimizations, we have assumed $40 \mathrm{~cm}$ as the maximal guide width for the longest guides, relying on information that guides of this width and matching slow frame-overlap choppers can be produced ${ }^{25,28}$. Should it be necessary to place stricter limits on the guide width this will affect the absolute flux values at some instruments ${ }^{26}$, but not the relative comparisons relevant for the present work. This statement is valid for most other design parameters.

For the long guides, no attempt has been made to avoid line-of-sight. The key issue is that bending of the guides, as known from traditional guide systems, would disturb the elliptical focusing properties ${ }^{26}$, whence a solution to this issue is more involved and was postponed to later studies $^{29}$.

An additional possibility to reduce the fast-neutron background would be to insert either a crystal filter or a heavy "straight-beam-block" in the middle of the guide, probably early in the guide ${ }^{30}$. Another possibility for guide design is the combination parabolic-straightparabolic, where the straight section can be curved. This combination transmists almost as well as an elliptical guide $^{24}$. 


\section{E. Optimization of instruments by simulation}

All present simulations were performed using the Monte-Carlo ray-tracing package McStas v. $1.12^{31}$, where the instrument designs were typically performed on individual computers, while the final optimization and data taking was performed on the computer cluster of the ESS Data-Management Center in Copenhagen. Typical runs used between $10^{8}$ and $10^{11}$ neutron rays, depending on the type of instrument.

Instruments were first simulated at the baseline time structure settings of $\tau=2 \mathrm{~ms}$ and $T=60 \mathrm{~ms}$. The instrument length and chopper settings were adjusted as to obtain a pre-determined instrumental resolution, while remaining above a certain length limit, relevant for the SANS and spin-echo instruments. Subsequently, the guide system of each instrument was optimized using a Figure-of-Merit (FoM) found from the time average flux, $\Psi(\lambda)$, on the sample position in the useful wavelength band, $\left[\lambda_{\min }, \lambda_{\max }\right]$ weighted by $V(\lambda)$ :

$$
\mathrm{FoM}=\int_{\lambda_{\min }}^{\lambda_{\max }} \Psi(\lambda) V(\lambda) d \lambda .
$$

Subsequently, the design of each of the 15 instruments was modified and optimized for each of 20 different time structure settings, in principle 300 optimizations and subsequent simulated data. In order to produce comparable simulations, all optimizations for a given instrument were restricted to have certain resolution characteristics. For spectrometers, this was given as $\delta \lambda / \lambda$ at the sample position for a certain value of $\lambda$. For diffractometers, this was given as a fixed $\delta \lambda / \lambda$ at the detector for a limited divergence matching this value, to obtain a certain low linewidth in the measured lattice spacing, $\delta d / d$, at a given scattering angle. For a few instrument types (spinecho spectrometer and SANS), the worst resolution was in all cases deemed "sufficient", so these instruments were not restricted by resolution requirements and were thus simulated at their constant (minimum) lengths.

Since it has been proposed to place triple-axis spectrometers at the long-pulsed ESS, we have included a cold and a thermal instrument in these comparisons. For a triple-axis spectrometer at a pulsed source, the time structure is useful only for filtering of background and higher-order harmonics. Hence, the instrument has identical FoM for all time structure settings, and we needed to simulate only one time structure for each of the two triple-axis spectrometers.

\section{RESULTS OF INSTRUMENT OPTIMIZATIONS}

We now present the results of our optimizations over the time structure range, as described above. To exemplify, we begin with the results for two individual instruments, before describing the combined results of the full instrument suite. Finally, we discuss the validity of our FoM approach.

\section{A. Simulation example 1: Cold Chopper Spectrometer}

Let us first consider the simulation of the cold-neutron chopper spectrometer, with a design similar to IN5 at ILL. In this present (simple) version of this instrument, the monochromatization is performed by the (full) length of the pulse, in combination with the opening time of fast choppers just before the sample, as illustrated in Fig. 2. The instrument length is determined by the pulse length, to fulfill a constant $\delta \lambda / \lambda=1.6 \%$ at $5 \AA$ wavelength. At the baseline time structure settings, the distance between the source and the fast chopper is $L=100 \mathrm{~m}$, and the useful band is $2.2 \AA$ wide (here chosen to be $3.9 \AA$ to $6.1 \AA$ ). This is described in detail in Ref. 5, where, however, a more simple guide system was used. Our results can thus be seen as an update of the previous publication.

The present cold chopper spectrometer uses an elliptical guide with quadratic cross section, which is $27.3 \mathrm{~cm}$ at its widest place. The guide focuses to the sample, which is defined to be $2 \times 2 \mathrm{~cm}^{2}$. The instrument uses the RRM scheme, as presented earlier This mode allows for each source pulse 9 monochromatic pulses on the sample, with a wavelength difference between neighbouring pulses of $0.25 \AA$, and $6 \mathrm{~ms}$ between pulses. In this way, the instrument reaches a combined monochromatic flux of $1.6 \cdot 10^{8} \mathrm{n} / \mathrm{s} / \mathrm{cm}^{2}$ for the wavelength band mentioned above, centered at $5 \AA$.

A shorter source pulse will allow for a shorter instrument; for pulses of $1.5 \mathrm{~ms}, 1.25 \mathrm{~ms}$, and $1.0 \mathrm{~ms}$, the instrument length becomes $80 \mathrm{~m}, 70 \mathrm{~m}$, and $60 \mathrm{~m}$, respectively. (The finite opening time of the monochromating choppers has the consequence that the $1.0 \mathrm{~ms}$ instrument is less than a factor two shorter than the $2.0 \mathrm{~ms}$ instrument.) A shorter instrument gives rise to a larger bandwidth and thus more neutrons on the sample (for constant time-average flux). For example, when going from $2 \mathrm{~ms}$ to $1 \mathrm{~ms}$ pulse length, the increase in FoM is more than $50 \%$, as seen in Table II. A rather similar gain is found from lowering the source frequency from $16 \frac{2}{3} \mathrm{~Hz}$ to $10 \mathrm{~Hz}$, also due to the larger useful bandwidth.

Due to the point-to-point-like focusing of an elliptial guide, most neutrons at the sample originate from the innermost $4 \times 4 \mathrm{~cm}^{2}$ of the moderator surface, as shown in Fig. 3. Therefore, it would be beneficial if neutrons were emitted preferentailly from the center of the moderator. A simulated hot spot with a factor 2.0 intensity gain over a circle of diameter $d=3 \mathrm{~cm}$ produces a gain in neutron flux at the sample of $30 \%$.

Taken at face value, the flux number obtained at the base time structure settings represents an impressive factor 200 gain over IN5. However, care should be taken when comparing these numbers. Firstly, the full gain is possible only if neutrons from all monochromatic pulses are equally useful to the actual experiment. Secondly, much of the flux increase comes from an increased divergence of neutrons in the elliptical guide system (compared to the straight/curved guide at IN5), and this part of the gain would be of value only to particular experi- 


\begin{tabular}{|l|rllll|r|}
\hline$T(\mathrm{~ms})$ & $\tau(\mathrm{ms})$ & 1.0 & 1.25 & 1.5 & 2.0 & $N$ \\
\hline $100(10 \mathrm{~Hz})$ & 2.39 & 2.24 & 2.05 & 1.67 & 15 \\
$80(12.5 \mathrm{~Hz})$ & 2.08 & 1.83 & 1.59 & 1.26 & 11 \\
$60(16.67 \mathrm{~Hz})$ & 1.72 & 1.48 & 1.29 & 1.00 & 9 \\
$50(20 \mathrm{~Hz})$ & 1.35 & 1.17 & 0.98 & 0.76 & 7 \\
$40(25 \mathrm{~Hz})$ & 0.91 & 0.81 & 0.68 & 0.56 & 5 \\
\hline
\end{tabular}

TABLE II. Relative Figure-of-Merit (FoM) values for the simulations of the IN5-like cold chopper spectrometer at ESS, under the assumption of constant time-average flux. Simulations are performed for 20 different settings of the time structure, $(T, \tau)$. The RRM scheme is parametrized by $N$, which indicates the number of possible monochromatic pulses at the sample per source pulse.

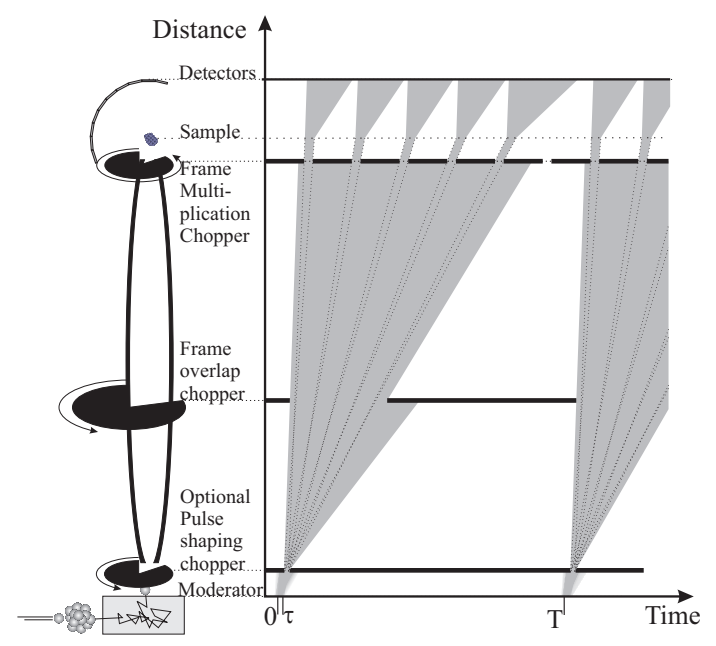

FIG. 2. (left) Sketch of the main elements of the cold chopper spectrometer. Picture is not to scale. (right) Time-offlight diagram illustrating the selection of neutron pulses by choppers, with the spectrometer running in RRM mode with $N=5$.

ment types. Hence, the mentioned gain is for this instrument a best case scenario, where a worst case scenario (collimating down to IN5 divergence and using only one RRM frame) would lead to a gain factor of "just" 5 .

\section{B. Simulation example 2: Long SANS instrument}

We now consider the longest of the three simulated cold-neutron small-angle scattering instruments. In analogy with the cold chopper spectrometer described above, the wavelength uncertainty is determined by the full pulse length, since the incoming wavelength is determined by the measured time-of-flight in the detector (assuming elastic scattering at the sample).

The length of the instrument is in practice determined by the $20 \mathrm{~m}$ long double-pinhole collimator section, combined with an initial $8 \mathrm{~m}$ of guide, which includes a kink to avoid direct line-of-sight. The source-sample distance
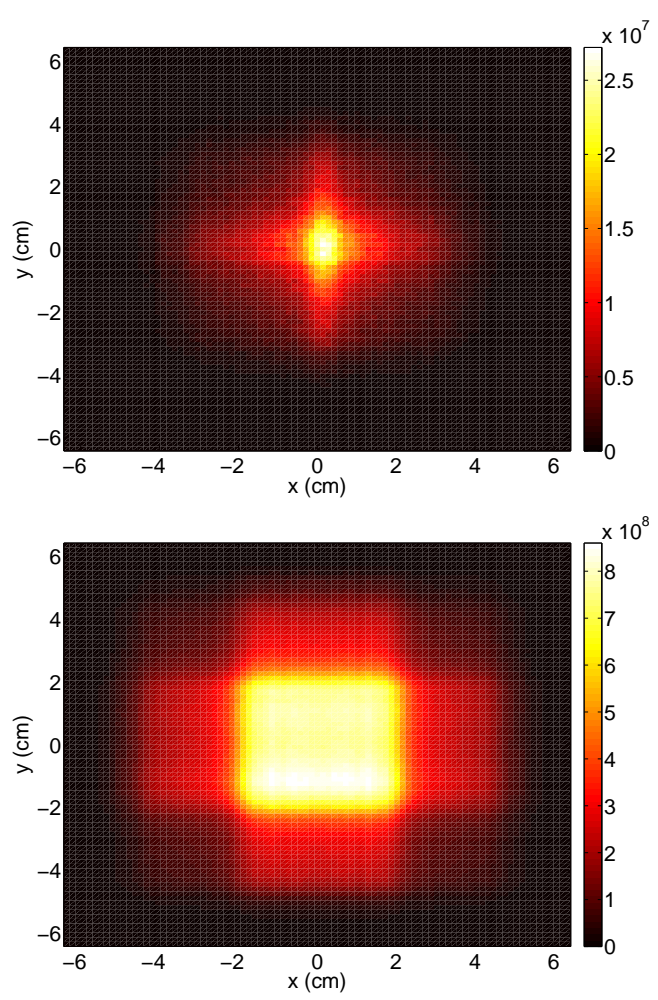

FIG. 3. (Color online). Simulated plots of the moderator surface showing the number of neutrons which reach the sample at the IN5-like chopper spectrometer. Top panel shows the situation with a $60 \mathrm{~m}$ guide $(\tau=1 \mathrm{~ms})$, while bottom panel shows the results for a $100 \mathrm{~m}$ guide $(\tau=2 \mathrm{~ms})$.

is thus always $28 \mathrm{~m}$, while the sample-detector distance can vary between $2 \mathrm{~m}$ and $20 \mathrm{~m}$. The relevant time-offlight length, $L$, thus varies between $30 \mathrm{~m}$ and $48 \mathrm{~m}$. At these lengths, the wavelength uncertainty at the SANS instrument at $\lambda=5 \AA$ and $\tau=2 \mathrm{~ms}$ is of the order $\delta \lambda / \lambda \approx 3-5 \%$, which is almost always "too good", since the double-pinhole collimation of $d_{1}=10.5 \mathrm{~mm}$ and $d_{2}=7.0 \mathrm{~mm}$ has the dominating contribution to the $q$-resolution.

The bandwidth of the instrument is rather large, of the order $8 \AA$ at the shortest detector setting. In combination with the large angular range covered at the detector, this allows a large $q$-range detected in the same setting. A sketch of the long SANS instrument and the corresponding wavelength band selection is found in Fig. 4.

In our optimizations, we have employed three settings of the collimation length and the sample-detector distance: $(2+2) \mathrm{m},(10+10) \mathrm{m}$, and $(20+20) \mathrm{m}$. The results presented are an average of the three results, each normalized by the result at the baseline setting. For the baseline setting, the instrument reaches neutron fluxes of $1.8 \cdot 10^{8} \mathrm{n} / \mathrm{s} / \mathrm{cm}^{2}, 9.0 \cdot 10^{5} \mathrm{n} / \mathrm{s} / \mathrm{cm}^{2}$, and $9.7 \cdot 10^{3} \mathrm{n} / \mathrm{s} / \mathrm{cm}^{2}$ for the three choices of distance, respectively, and the wavelength band centered around $10 \AA$.

A shorter source pulse will give better wavelength reso- 


\begin{tabular}{|l|rllll|}
\hline$T(\mathrm{~ms})$ & $\tau(\mathrm{ms})$ & 1.0 & 1.25 & 1.5 & 2.0 \\
\hline $100(10 \mathrm{~Hz})$ & 1.69 & 1.69 & 1.69 & 1.69 \\
$80(12.5 \mathrm{~Hz})$ & 1.34 & 1.34 & 1.34 & 1.34 \\
$60(16.67 \mathrm{~Hz})$ & 1.00 & 1.00 & 1.00 & 1.00 \\
$50(20 \mathrm{~Hz})$ & 0.83 & 0.83 & 0.83 & 0.83 \\
$40(25 \mathrm{~Hz})$ & 0.67 & 0.67 & 0.67 & 0.67 \\
\hline
\end{tabular}

TABLE III. Relative Figure-of-Merit (FoM) values for the simulations of the long SANS instrument at ESS under the assumption of constant time-average flux. Simulations are performed for 20 different settings of the time structure, $(T, \tau)$.

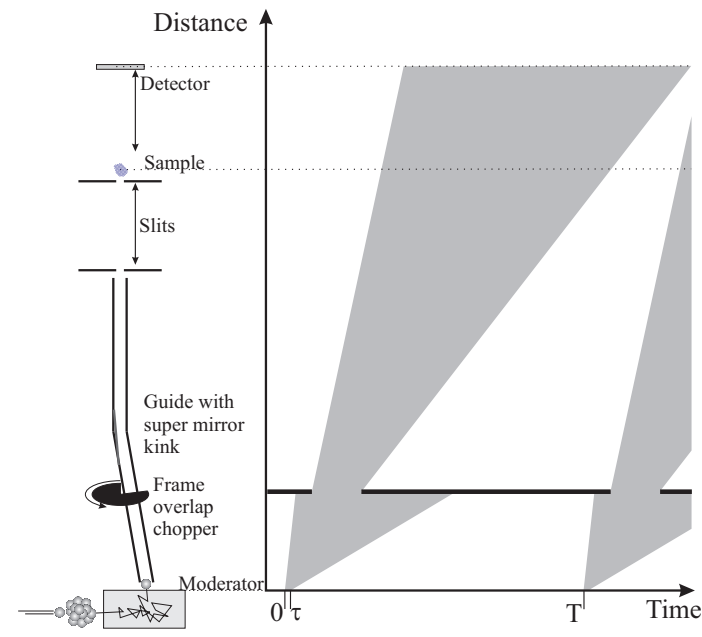

FIG. 4. (left) Sketch of the main elements of the long SANS instrument. Details are not to scale. (right) Time-of-flight diagram illustrating the selection of wavelength band by choppers, with the spectrometer running in the $(20+20) \mathrm{m}$ setting.

lution, but the instrument cannot be shortened due to the kink and the collimation section. Therefore, this gives no gain in bandwidth (or integrated flux), but a small improvement in $q$-resolution. If, on the other hand, the source frequency is lowered, e.g. to $10 \mathrm{~Hz}$, at constant time-average flux, the instrument will benefit from an increase in useful bandwidth and hence the FoM will increase. All FoM data are displayed in Table III.

At the longest collimation length, all neutrons at the sample originate from a circle of $d \approx 2.5 \mathrm{~cm}$ at the center of the moderator surface. This effect is less pronounced at the shorter collimation lengths. This is illustrated in Fig. 5. On average, a simulated hot spot with a factor 2.0 intensity gain over a circle of diameter $d=3 \mathrm{~cm}$ produces a gain in neutron flux at the sample of $73 \%$.

\section{Optimization of the full instrument suite}

After the optimization procedures, we record the resulting values of wavelength, bandwidth, flux at sample position, and FoM for each instrument and time struc-
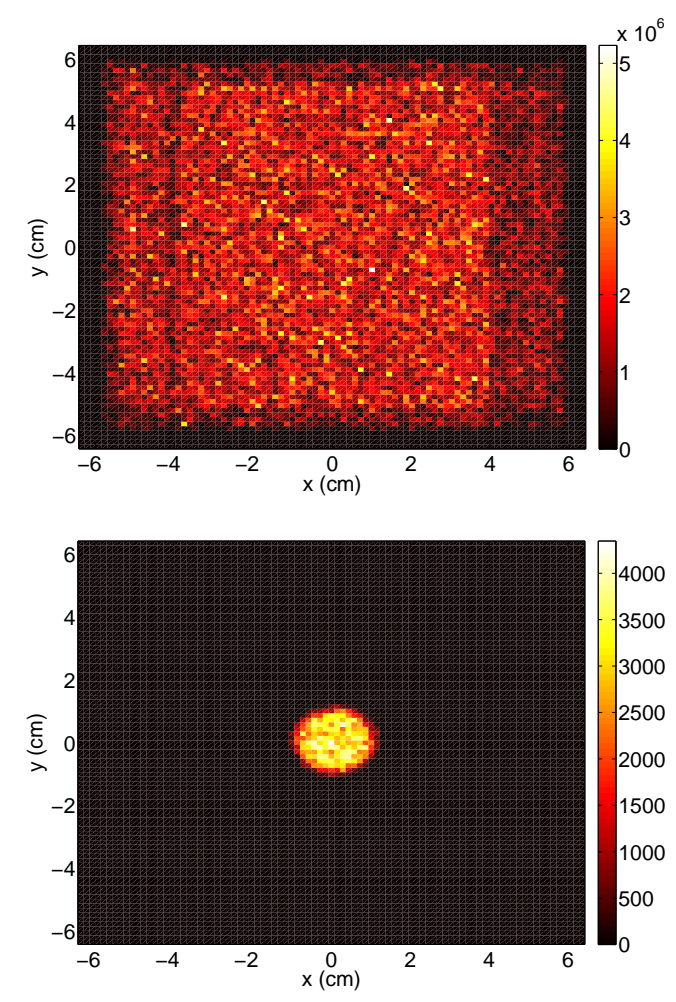

FIG. 5. (Color online). Simulated plots of the moderator surface showing the number of neutrons which reach the sample for the $20 \mathrm{~m}$ SANS instrument. The results are valid for any time structure. (top) data for $2 \mathrm{~m}$ collimator-detector setting; (bottom) data for $20 \mathrm{~m}$ collimator-detector setting.

ture setting. The results of the individual simulations are in general similar to the simulation results of the chopper spectrometer and the small-angle instrument shown above. (Results can be found from 4.) The obtained values of FoM have been normalized to the baseline setting of $T=60 \mathrm{~ms}$ and $\tau=2 \mathrm{~ms}$.

For constant time-average flux, almost all instruments perform better towards the upper left-hand corner of the performance matrix. This is as expected, since i) a longer $T$ will allow for a larger useful wavelength band, $\Delta \lambda$, and ii) a smaller $\tau$ will either iia) allow $L$ to be smaller, giving an increased $\Delta \lambda$, or iib) allow a higher fraction of the total flux through the pulse-defining choppers.

In contrast, for the constant-peak-flux scenario, most instruments perform better towards the lower right corner of the performance matrix. This is most simply explained by the fact that here, more neutrons are produced in total, overcompensating the advantages of short pulses and low frequencies mentioned above.

To perform a global comparison of the different time structure settings, we use the relative instrument performances for each instrument. A simple arithmetic mean value has been used, since no decision on the relative importance of instruments has been taken. The results for the average performances are listed in Tables IV 


\begin{tabular}{|l|rllll|}
\hline$T(\mathrm{~ms})$ & $\tau(\mathrm{ms})$ & 1.0 & 1.25 & 1.5 & 2.0 \\
\hline $100(10 \mathrm{~Hz})$ & 2.07 & 1.81 & 1.67 & 1.37 \\
$80(12.5 \mathrm{~Hz})$ & 1.89 & 1.66 & 1.55 & 1.19 \\
$60(16.67 \mathrm{~Hz})$ & 1.62 & 1.42 & 1.24 & 1.00 \\
$50(20 \mathrm{~Hz})$ & 1.53 & 1.27 & 1.09 & 0.88 \\
$40(25 \mathrm{~Hz})$ & 1.20 & 1.05 & 0.90 & 0.73 \\
\hline
\end{tabular}

TABLE IV. Average relative Figure-of-Merit for the generic ESS instrument suite at different time structures, under the assumption of constant time-average flux.

\begin{tabular}{|l|rllll|}
\hline$T(\mathrm{~ms})$ & $\tau(\mathrm{ms})$ & 1.0 & 1.25 & 1.5 & 2.0 \\
\hline $100(10 \mathrm{~Hz})$ & 0.62 & 0.68 & 0.75 & 0.82 \\
$80(12.5 \mathrm{~Hz})$ & 0.71 & 0.78 & 0.87 & 0.89 \\
$60(16.67 \mathrm{~Hz})$ & 0.81 & 0.89 & 0.93 & 1.00 \\
$50(20 \mathrm{~Hz})$ & 0.92 & 0.95 & 0.98 & 1.05 \\
$40(25 \mathrm{~Hz})$ & 0.90 & 0.98 & 1.01 & 1.09 \\
\hline
\end{tabular}

TABLE V. Average relative Figure-of-Merit for the generic ESS instrument suite at different time structures, under the assumption of constant peak flux.

and $\mathrm{V}$ for the constant-time-average-flux and constantpeak-flux scenarios, respectively.

We see that the effect of shortening the pulse from $2.0 \mathrm{~ms}$ to $1.0 \mathrm{~ms}$ is typically around $60 \%$ increase at constant time-average flux - or around $20 \%$ decrease at constant peak flux. Likewise, the effect of going from $20 \mathrm{~Hz}$ to $10 \mathrm{~Hz}$ is around a $50 \%$ increase at constant time-average flux - or around $30 \%$ decrease at constant

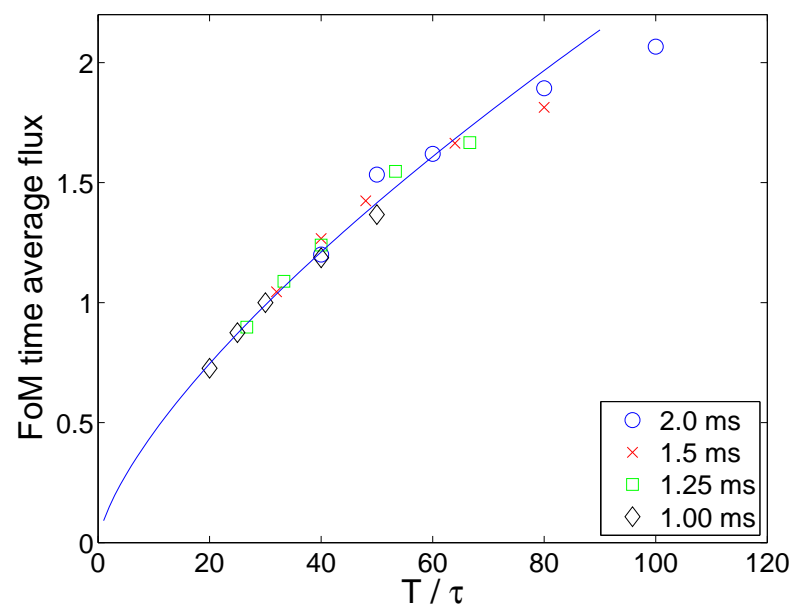

FIG. 6. (Color online). Average Figure-of-Merit for the generic ESS instrument suite at different time structures, plotted as a function of the inverse source duty cycle, under the assumption of constant time-average flux. Diamonds, squares, crosses, and circles represent pulse lengths of 2.0, 1.5, 1.25 , and $1.0 \mathrm{~ms}$, respectively. The solid line is a fit to the power law (6), as explained in the text. peak flux.

The data for constant time-average flux is shown as a function of the inverse source duty cycle, $T / \tau$, in Figure 6 . It is seen that, except for the very smallest duty cycles, the data fall almost on a common curve, which can be approximately described by

$\mathrm{FoM} \approx \Psi_{\text {peak }}\left(\frac{\tau}{T}\right)^{\alpha}=\Psi_{\text {peak }}^{1-\alpha} \Psi_{\text {time av }}^{\alpha}=\Psi_{\text {time av }}\left(\frac{T}{\tau}\right)^{1-\alpha}$

with $\alpha=0.30$. This value confirms the trivial result that the long-pulse source of the ESS is intermediate in nature between a short-pulse source and a continuous source. Instruments at short-pulse sources aim to be optimised to benefit from the peak flux, while instruments at steady-state sources are optimised to benefit from the time-average flux. The exact value of the exponent will clearly depend on the chosen instrument suite, but it is interesting to note firstly that the instrument performance scales more closely with the peak flux than with the time-average flux. Secondly, equation (6) predicts that the global instrument performance depends only on the time-average flux and duty cycle. If both are kept constant, e.g. by changing $T$ and $\tau$ simultaneously, the instrument performance should be independent of the time structure.

Our results can be seen as a natural continuation of the discussion on the merits of long-pulse sources, initiated in Ref. ${ }^{14}$. While the previous work has used basic, yet solid approximation to advocate the usefulness of long neutron pulses, our results serves quantify this usefulness, including important features like moderator spectrum and instrument-specific details over the instrument suite.

\section{Considerations beyond a simple Figure-of-Merit}

The analysis above is based on the assumption that it is possible to reduce the full scientific usefulness of a facility into one single number, the FoM, and to express its variation by essentially one parameter, the duty ratio $\tau / T$, as illustrated in Figure 6. This assumption shares one problem with most numerical optimization work: Details that cannot be compressed into the FoM are easily overlooked. For this reason, we will look more into some of these details. To simplify the argument, we will consider the effect on the instrument performance under the condition that the duty cycle $\tau / T$ is unchanged. The effect of varying the time structure under this boundary condition depends rather sensitively on the type of instrument.

- SANS, reflectometry and spin-echo instruments will benefit from the increased wavelength range which a longer repetition period will give them. Their performance will not suffer significantly from the degraded wavelength resolution, which an associated increase in pulse length would give. Any 
increase in bandwidth translates directly into improved performance.

- Crystal-monochromator instruments, such as triple-axis spectrometers, do not make much use of the source time structure at all. In these cases, only the time-average flux counts. The time structure has little or no effect.

- Chopper spectrometers, or other instruments that may employ RRM, have a weak preference for shorter repetition periods. These instruments use the RRM to compensate for the fact that their preferred repetition frequency is higher than the source frequency. Increasing the source frequency reduces the need for RRM and makes their data-collection strategy more similar to existing instruments and simplifies the data analysis.

- Very high-resolution instruments, such as backscattering spectrometers and high-resolution diffraction also have a preference for shorter repetition periods. These instruments cut out only a small fraction of the pulse length to achieve the desired resolution and do not benefit significantly from the increased wavelength range offered by an increase in repetition period.

Overall, it seems clear that an increase in pulse length will translate into an increase in the average length of the instruments, which will result in increased costs for guides and shielding along the guides. On the other hand, with modern ballistic-type guides, the transport of neutrons represents no essential problem ${ }^{24}$, while the instrument space becomes less restricted at the same time as the general background level decreases. In addition, certain combinations of $T$ and $\tau$ may result in instruments with lengths which allow them to be grouped together in common instrument halls, rather than requiring separate buildings. In such a scenario, the cost savings associated with the reduction in the number of instrument buildings could cancel out the cost increase of the longer guides, as well as providing other benefits in terms of upgradeability and flexibility.

\section{E. Optimizing the moderator parameters}

The design and simulation of the target/moderator is much more computationally demanding than that of the instruments. Hence, one aim of the instrument simulations has been to assist the moderator optimizations towards an improved functionality of the full ESS. We here describe the results obtained in this direction.

Often, the figure-of-merit in moderator optimizations is the number of neutrons produced, possibly in a given wavelength interval and for a given moderator size. However, the moderator simulations produce more detailed information than this. The result of each simulation is given as a history of neutron events, each event having 6 parameters: position at moderator surface $(\mathbf{r})$, time of emittance $(t)$, wavelength $(\lambda)$, and moderator emission angle $(\boldsymbol{\eta})$. By means of instrument simulations it was found possible to represent the transmission probability of a neutron from moderator surface to sample as

$$
T(\mathbf{r}, \lambda) \approx T_{r}(\mathbf{r}) T_{\lambda}(\lambda) .
$$

Here, the dependence on divergence has been integrated out, since the moderator flux (even with complex geometries) is expected to vary insignificantly over the rather small solid angle of the guide entry, Furthermore, we have neglected the emission time, which corresponds to ignoring the tails from the moderators. For a total target/moderator optimization, the figure-of-merit to optimize is thus for each of the moderators (e.g. a cold and a thermal):

$$
\begin{aligned}
& \mathrm{FoM}_{\bmod }=\sum_{j} W_{j} \int N(\mathbf{r}, \lambda, t, \boldsymbol{\eta}) V_{j}(\lambda) \\
& \times T_{j, r}(\mathbf{r}) T_{j, \lambda}(\lambda) d^{2} \mathbf{r} d \lambda d^{2} \boldsymbol{\eta} d t
\end{aligned}
$$

where the summation label, $j$, represents the instruments at the moderator, $W_{j}$ is a normalization and weighting constant for each instrument, $N$ is the simulated density of neutrons from the moderator, and $V_{j}(\lambda)$ is given in (1).

We have for each instrument calculated the spatial transmission function, $T_{r}(\mathbf{r})$, as shown in the examples above, and listed in Ref. 4. The results show that for most instruments, the transmission peaks strongly in a $3-5 \mathrm{~cm}$ diameter circle (or square) in the center of the moderator. This effect results for some instruments from the use of elliptical guides, for others from using tight collimation and straight guides. Thus, it can for these simple guide systems be advantageous to concentrate the flux in a hot spot, while the size of the emitting part of the moderator can be limited, e.g. by reflectors. In this way, it should be possible to simultaneously increase the useful neutron flux and decrease the emission of fast neutrons. For each instrument, we have calculated the effect of producing a circular, $3 \mathrm{~cm}$ diameter, hot spot with $100 \%$ higher emittance - while maintaining the total emittance of the moderator. This set-up is close to what was presented in Ref. ${ }^{32}$. For most instruments, the gain factor of such a hot spot is around $30 \%$, while few instruments show a full $100 \%$ gain.

It should be added that more elaborate guide systems, in particular an optimized guide extraction system for instruments with a pulse shaping chopper close to the moderator, will modify this picture. This problem will be addressed by further simulation work ${ }^{29}$.

\section{v. CONCLUSION}

We have performed a series of systematic ray-tracing simulations of the performance of a generic instrument 
suite for the ESS. These simulations were carried out for a large number of time structure settings, for constant, typical instrument resolutions. The performance parameters were found to increase with increasing peak flux, as well as with increasing time-average flux, while varying only weakly with the details of the time structure.

The variation with time-average and peak flux can be expressed as FoM $\propto \Psi_{\text {peak }}(\tau / T)^{\alpha}$, with $\alpha=0.30$. If both the peak flux and the duty cycle are kept constant, the average instrument performance is largely independent of pulse-length or frequency, within the frequency range of the current study.

Since most instruments use tight collimations or (elliptical) focusing guides, most neutrons hitting the sample stem from a central part of the moderator of a diameter $3-5 \mathrm{~cm}$. We suggest to use this knowledge for the optimization of the moderator design, in particular by considering "hot spots" at the moderator. However, this can be finalized only when the guide systems of the instruments are designed.

\section{A. Implications for design of long-pulsed sources, e.g. ESS}

As part of the study which resulted in the decision to fix the time structure of the ESS to $\tau=2.86 \mathrm{~ms}$ and $T=71 \mathrm{~ms}(f=14 \mathrm{~Hz})$, two boundary conditions were considered: 1) the time-average power is planned to be 5 MW. 2) the peak accelerator current cannot exceed $50 \mathrm{~mA}$. The $5 \mathrm{MW}$ number is judged to be important, so as to at least match the best existing instruments over the largest possible range. The limitation on the peak current results from a judgement, based on the experience of the SNS linear accelerator, as to the optimal compromise between performance, reliability and cost. In the interest of maximizing the instrument performance, it is clearly advantageous to push for the highest peak flux which the accelerator and target assembly can provide. We can therefore consider the $50 \mathrm{~mA}$ peak current as our specification, rather than an upper limit. These boundary conditions reduce the number of degrees of freedom when choosing $\tau$ and $T$ from 2 to 1 , as follows. The peak power on target is given by the product of the peak current and proton energy of $2.5 \mathrm{GeV}$. At $50 \mathrm{~mA}$ peak current, the instantaneous power is $125 \mathrm{MW}$. In order to achieve a time-average power of $5 \mathrm{MW}$, the source therefore needs to operate at a duty cycle $\tau / T$ of $5 \mathrm{MW} / 125 \mathrm{MW}=1 / 25$, as a direct consequence of our two boundary conditions. If we set the repetition period to $100 \mathrm{~ms}(10 \mathrm{~Hz})$, the pulse length will be $4 \mathrm{~ms}$. At $T=50 \mathrm{~ms}$ repetition period $(f=20 \mathrm{~Hz})$, the pulse length is $2 \mathrm{~ms}$. The range of $\tau$ and $T$ covered in the present study only overlaps partially with the duty cycle $\tau / T=1 / 25$. In order to study the instrument performance over the $10-25 \mathrm{~Hz}$ frequency range, while maintaining a duty cycle of $1 / 25$, we extrapolate based on the data in Tables IV and V and eq. (6) that the performance of the instrument suite does not depend upon the value of the source frequency.
In general, our results imply that factors other than the flux-related FoM used here should be decisive when determining the time structure for a long-pulse spallation source. For the case of ESS, the time structure has now been locked to $\tau=2.86 \mathrm{~ms}$ and $f=14 \mathrm{~Hz}$, as the best compromise between performance, reliability and cost. The detailed considerations are outside the scope of this article.

\section{B. Further design and optimization of ESS instruments}

The instrument design work for ESS is currently taking place in a setting which is very different from when the design work described in the present paper was taking place. A large number of the neutron laboratories and university groups working in neutron scattering in Europe are now engaged in the process of designing instruments for the ESS and the number is still increasing. About 40 different concepts for instruments are currently being optimized, some pursued by researchers in partner countries and some by ESS instrument scientists. A subset of these concepts has been assembled into a reference suite of instruments which is described in the ESS Technical Design Report ${ }^{33}$. The reference suite has been chosen to maximize the scientific impact of the ESS by addressing a broad science case while in each case being fully optimized to benefit from the natural strengths of the long-pulse concept. The choice of instruments to be built at the ESS will take place as a staged process in consultation with the European scientific community and will result in the reference suite gradually evolving into the actual instrument which will be available at the ESS.

\section{ACKNOWLEDGEMENTS}

First of all we are strongly indepted to Ch. Vettier for initiating this project and to D. Argyriou for keeping up the urgency of the simulations. We would further like to thank the ESSS SAG, the ESS SAC, and H.M. Rønnow for illuminating discussions.

This project was supported by the Danish, Norwegian, and German contributions to the Design Update phase of the ESS. J.O. Birk was supported by PSI and the Danish Research Council through the graduate school C:O:N:T.

\section{REFERENCES}

\footnotetext{
${ }^{1}$ See the home page for the ESS project: www.ess-scandinavia.eu ${ }^{2}$ D. Richter (ed.), The ESS Project (Jülich Research Center 2002), Vol I

${ }^{3}$ D. Richter (ed.), The ESS Project (Jülich Research Center 2002), Vol IV
} 
${ }^{4}$ See the home page sim.esss.dk

${ }^{5}$ K. Lefmann, H. Schober, and F. Mezei, Meas. Sci. Techn., 19, 034025 (2008)

${ }^{6}$ K. Lefmann, U. Filges, F. Treue, J.J.K. Kirkensgård, B. Plesner, K.S. Hansen, and K.H. Klenø, Nucl. Instr. Meth. A 634, S1 (2011)

${ }^{7}$ M. Sales, S.L. Holm, K. Lieutenant, and K. Lefmann, J. Phys. Soc. Jpn., 80, SB018 (2011)

${ }^{8}$ K.H. Klen $\varnothing$ and K. Lefmann, J. Phys. Soc. Jpn., 80, SB004 (2011)

${ }^{9}$ K.H. Klen $\varnothing$, P.K. Willendrup, E. Knudsen, and K. Lefmann, Nucl. Instr. Meth. A 634, S100 (2011)

${ }^{10}$ K.H. Klenø, S. Kynde, G. Nagy, K. mortensen, K. Lefmann, J. Kohlbrecher, and L. Arleth, A Compact Time-of-Flight SANS Instrument Optimised for Measurements of Small Sample Volumes at the European Spallation Source, submitted to J. Appl. Cryst. (2013)

${ }^{11}$ J.O. Birk et al., Simulations of the inverse-geometry time-offlight spectrometer CAMEA forthe European Spallation Source, in preparation (2013)

12 A. Vickery et al., Comparing thermal spectrometers for a longpulse spallation source, in preparation (2013)

${ }^{13}$ R.K. Crawford et al., Report of the Long-pulse Neutron Instrumentation Workshop, Frascati, August 2009 (2010)

${ }^{14}$ F. Mezei, Journal of Neutron Research 6, 3 (1997)

${ }^{15} \mathrm{G}$. Zsigmond et al., Appl. Phys. A 74, S224 (2002)

${ }^{16}$ H. Schober et al., Nucl. Instr. Meth. A 589, 34 (2008)

${ }^{17} \mathrm{~K}$. Lefmann et al., Report from the Ven 2008 meeting (unpublished)
${ }^{18}$ K. Lieutenant and F. Mezei, J. Neutron Res. 14, 177 (2006)

${ }^{19}$ M. Russina and F. Mezei, Proc. SPIE (Int. Soc. f. Opt. Eng.) 4785, 24 (2002)

${ }^{20}$ F. Mezei, M. Russina, and S. Schorr, Physica B, 276, 128 (2000)

${ }^{21}$ M. Russina and F. Mezei, Nucl. Instr. Meth. A 604, 624 (2009)

${ }^{22}$ M. Nokamura et al., J. Phys. Soc. Jpn. 78, 093002 (2009)

${ }^{23} \mathrm{~F}$. Mezei, ESS reference moderator characteristics for generic instrument performance evaluation, (ESS Project, Jülich, December 2000); see also http://rencurel.essworkshop.org/documents/ess_itg/ESS-mode

${ }^{24}$ K.H. Klen $\varnothing$, K. Lieutenant, K.H. Andersen, and K. Lefmann, Nucl. Instr. Meth. A 696, 75 (2012)

${ }^{25}$ SwissNeutronics A.G., personal communication; see also www.swissneutronics.ch

${ }^{26}$ K.H. Klenø et al., Effects of non-idealities in long neutron guides, in preparation (2013)

${ }^{27}$ P. Allenspach, P. Böni, and K. Lefmann, Proceedings of SPIE 4509 (2001)

${ }^{28}$ Mirrotron Ltd., personal communication; see also www.mirrotron.kfkipark.hu

${ }^{29}$ M. Bertelsen et al., Exploring performance of neutron guide systems using pinhole beam extraction, sumitted to Nucl. Instr. Meth. A (2013)

${ }^{30} \mathrm{U}$. Filges, personal communication

${ }^{31}$ K. Lefmann and K. Nielsen, Neutron News 10/3, 20 (1999); see also www.mcstas.org

${ }^{32}$ Y. Kiyanagi et al., Proceedings of the ICANS conference, PSI report (2010)

${ }^{33}$ S. Peggs (ed.), ESS Technical Design Report (European Spallation Source ESS AB 2013) 